\title{
TU/e EmonOWEN

\section{The influence of triplet exciton and charge transfer energy alignment on organic magnetoresistance}

\section{Citation for published version (APA):}

Janssen, P., Wouters, S. H. W., Cox, M., \& Koopmans, B. (2014). The influence of triplet exciton and charge transfer energy alignment on organic magnetoresistance. Organic Electronics, 15, 743-750.

https://doi.org/10.1016/j.orgel.2014.01.010

DOI:

10.1016/j.orgel.2014.01.010

Document status and date:

Published: 01/01/2014

\section{Document Version:}

Publisher's PDF, also known as Version of Record (includes final page, issue and volume numbers)

\section{Please check the document version of this publication:}

- A submitted manuscript is the version of the article upon submission and before peer-review. There can be important differences between the submitted version and the official published version of record. People interested in the research are advised to contact the author for the final version of the publication, or visit the $\mathrm{DOI}$ to the publisher's website.

- The final author version and the galley proof are versions of the publication after peer review.

- The final published version features the final layout of the paper including the volume, issue and page numbers.

Link to publication

\section{General rights}

Copyright and moral rights for the publications made accessible in the public portal are retained by the authors and/or other copyright owners and it is a condition of accessing publications that users recognise and abide by the legal requirements associated with these rights.

- Users may download and print one copy of any publication from the public portal for the purpose of private study or research.

- You may not further distribute the material or use it for any profit-making activity or commercial gain

- You may freely distribute the URL identifying the publication in the public portal.

If the publication is distributed under the terms of Article 25fa of the Dutch Copyright Act, indicated by the "Taverne" license above, please follow below link for the End User Agreement:

www.tue.nl/taverne

Take down policy

If you believe that this document breaches copyright please contact us at:

openaccess@tue.nl

providing details and we will investigate your claim. 


\title{
The influence of the triplet exciton and charge transfer state energy alignment on organic magnetoresistance
}

\author{
Paul Janssen, Steinar H.W. Wouters, Matthijs Cox, Bert Koopmans* \\ Department of Applied Physics, Center for NanoMaterials (cNM), Eindhoven University of Technology, PO Box 513,5600 MB Eindhoven, The Netherlands
}

\section{A R T I C L E I N F O}

\section{Article history:}

Received 4 July 2013

Received in revised form 10 January 2014

Accepted 12 January 2014

Available online 24 January 2014

\section{Keywords:}

Organic magnetoresistance

Magnetic field effects

Magnetoconductance

Organic semiconductors

Triplet energy

Doping

\begin{abstract}
A B S T R A C T
Recently, it was discovered that the current through an organic semiconductor, sandwiched between two non-magnetic electrodes, can be changed significantly (up to 25\%) by applying a small (a few millitesla) magnetic field. At present, the microscopic mechanisms underlying this so-called organic magnetoresistance (OMAR) are intensively being debated. One of the mechanisms which can successfully describe the magnetic field effects on the current in pristine organic semiconductor devices uses the reactions of triplet excitons and polarons. Here, we present a proof of concept study in which we tune these interactions in the device by deliberately doping our devices with fullerene, creating additional charge transfer states (CTS). By engineering devices with different energetic alignments of the CTS and triplet exciton, we can influence the triplet exciton density in the device. We correlate pronounced changes in the magnetic field effect magnitude and lineshape to the energy of the CTS with respect to the triplet exciton.
\end{abstract}

(c) 2014 Elsevier B.V. All rights reserved.

\section{Introduction}

The discovery of surprisingly large, room temperature, magnetoresistance effects in organic semiconductor devices without any ferromagnetic components has puzzled the young field of organic spintronics during the past decade [1-3]. The effect is often referred to as organic magnetoresistance (OMAR). The possibility to obtain large (up to $25 \%$ ) changes in the current at room temperature, while applying only small (a few millitesla) magnetic fields, makes this effect interesting for future applications and, moreover, from a scientific point of view. Studying OMAR provides a deeper fundamental understanding of spin-physics and charge transport in organic semiconductor devices.

All contemporary models explaining OMAR rely on magnetic field dependent reactions of the spin carrying particles, where a magnetic field suppresses the spin mixing of the particle pairs prior to the reaction. There is, however, an active debate about the nature of the particles

\footnotetext{
* Corresponding author.

E-mail address: b.koopmans@tue.nl (B. Koopmans).
}

(e.g. electrons, holes, bipolarons, excitons or trions) and the exact underlying mechanism influencing the current [4-13]. Currently, the possible mechanisms can be divided into two main categories: (i) mechanisms which only need one type of charge carrier or (ii) mechanisms which need both charge carriers. In the first class, the so-called bipolaron mechanism [6] has been successfully applied to explain magnetic field effects in unipolar devices $[8,14,15]$. In the second class, we can distinguish between models which describe the reactions of polarons with opposite charge into excitons [4] and models which rely on the spin dependent reactions of triplet excitons with polarons $[5,12]$. Besides the microscopic models, it is also important to be aware of the highly non-trivial role played by the device physics in order to explain experimentally observed magnetic field effects (MFEs) on the current [16,17]. Very recently, we have shown that the dominant mechanism for OMAR depends on the exact material choice and operating conditions of the device [15] and that the MFE in pristine organic semiconductor devices are predominantly governed by the reactions of (trapped) triplet excitons and polarons [12]. 
To study the underlying mechanism of triplet-polaron interactions, an exquisite control over the triplet exciton density is desired. In line with earlier work $[8,15]$, we propose polymer-fullerene blends as an ideal model system. We study the influence of triplet excitons on OMAR by introducing a low concentration of fullerene in the polymer devices. This creates additional charge transfer states (CTS) by which the number of triplet excitons can be increased, stabilized or decreased, depending on the relative energetic alignment of the CTS with respect to the triplet exciton [18]. Different energetic alignments can be engineered by choosing the right material combinations [19].

\section{Theory}

\subsection{Magnetic field effects}

As introduced in the foregoing, mixing of spin states plays a crucial role in OMAR. In this section we will show how spin mixing can lead to magnetic field effects on the current. We start our discussion with MFEs in pristine polymer devices. Fig. 1 presents an energy diagram of the relevant spin dependent transitions from free charges to the ground state. Free charges can form precursor pairs in a singlet ${ }^{1}()$ or triplet ${ }^{3}()$ configuration. Within the precursor pairs the separation between the two carriers is such that exchange interactions are still negligible. This enables hyperfine fields, originating from the hydrogen nuclei in the organic material, to mix the singlet and triplet precursor pairs. An external magnetic field suppresses this mixing. From the precursor pair state, the electron-hole pair can recombine into a singlet $(\mathrm{S})$ or triplet $(\mathrm{T})$ exciton and thereafter to the ground state.

In the absence of a magnetic field (Fig. 1b), spin mixing increases the number of triplet excitons if the formation rate of triplets is larger than that of singlets [11]. This is indicated by process ISC $_{\text {eh }}$ in Fig. $1 \mathrm{~b}$. Triplet excitons can decrease the current by interacting with free charge carriers $[5,12]$. Increasing the magnetic field (Fig. 1a) reduces the triplet formation and thus reduces the triplet-polaron interactions and thereby increases the total current, leading to a positive MC. This magnetic field effect, which is governed by the hyperfine field strength experienced by the precursor pair, will be referred to as a low field effect (LFE) and has a linewidth of a few millitesla.
The LFE in the pristine polymer device can be accompanied by a distinct magnetic field effect at ultra-small field scales (USFE, typically $\leqslant \mathrm{mT}$ ) as has recently been demonstrated $[11,20]$. Moreover, the triplet-polaron interaction is also magnetic field dependent, leading to so-called high field effects (HFE) occurring at field scales on the order of the zero-field splitting (ZFS, typically $100 \mathrm{mT}$ ) of the triplet exciton. When describing the triplet-polaron interactions using a mechanism where (trapped) triplet excitons temporarily capture free polarons into meta-stable trions, as proposed by Cox et al. then this HFE results in a positive MC.

\subsection{Introducing additional states}

To further investigate the underlying mechanism of triplet-polaron interactions, we are going to control the triplet exciton density by introducing additional states in the energy diagram. Hereby, the number of triplet excitons can be increased, stabilized or decreased. As introduced, this can be accomplished by adding fullerene to the polymer devices. At low concentration, the fullerene effectively quenches singlet excitons into weakly bound, spatially separated charge transfer states (CTS), where the hole resides on the polymer and the electron on the fullerene. The effect of fullerene on the triplet excitons depends on the energetic alignment of the CTS and triplet exciton, where three different kinds of devices can be distinguished.

First, in device type I, where the triplet exciton lies higher in energy than the CTS, the triplet exciton will also be effectively transferred into a CTS in a triplet configuration, as depicted by process $\mathrm{T} \rightarrow$ CTS in the top panel of Fig. 2. When both states are energetically aligned, the CTS and triplet exciton are in equilibrium and an increase in the number of triplet excitons will be stabilized by the CTS, as indicated by process $\mathrm{T} \leftrightarrow \mathrm{CTS}$ in the middle panel of Fig. 2. Finally, in device type II, the triplet exciton lies lower in energy than the CTS and the number of triplet excitons can increase by a back-transfer from the triplet CTS, as shown by process CTS $\rightarrow \mathrm{T}$ in bottom panel of Fig.2.

The introduction of a CTS can have profound effects on the MFEs, since the number of triplet excitons, ultimately responsible for the magnetic field effect on the current, can be changed significantly in this manner. We will now briefly explain the MFEs. In device type I, CTS decrease the

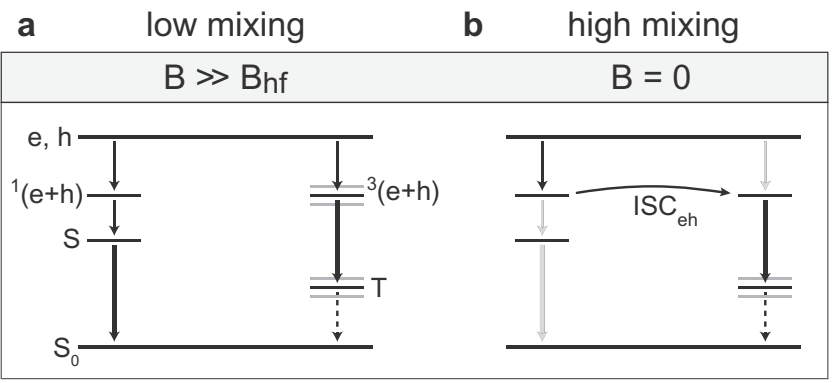

Fig. 1. Energy diagram of relevant spin dependent transitions (indicated by the arrows) from free charges to the ground state in a pristine device. (a) In a large external applied magnetic field, free charges can form precursor pairs in a singlet ${ }^{1}(e+h)$ or triplet ${ }^{3}(e+h)$ configuration. From this pair state, the precursor pair can recombine into a singlet $(\mathrm{S})$ or triplet $(\mathrm{T})$ exciton. (b) Without a magnetic field, singlet and triplet precursor pairs can mix due to hyperfine interactions, as indicated by transition ISC $_{\mathrm{eh}}$. 


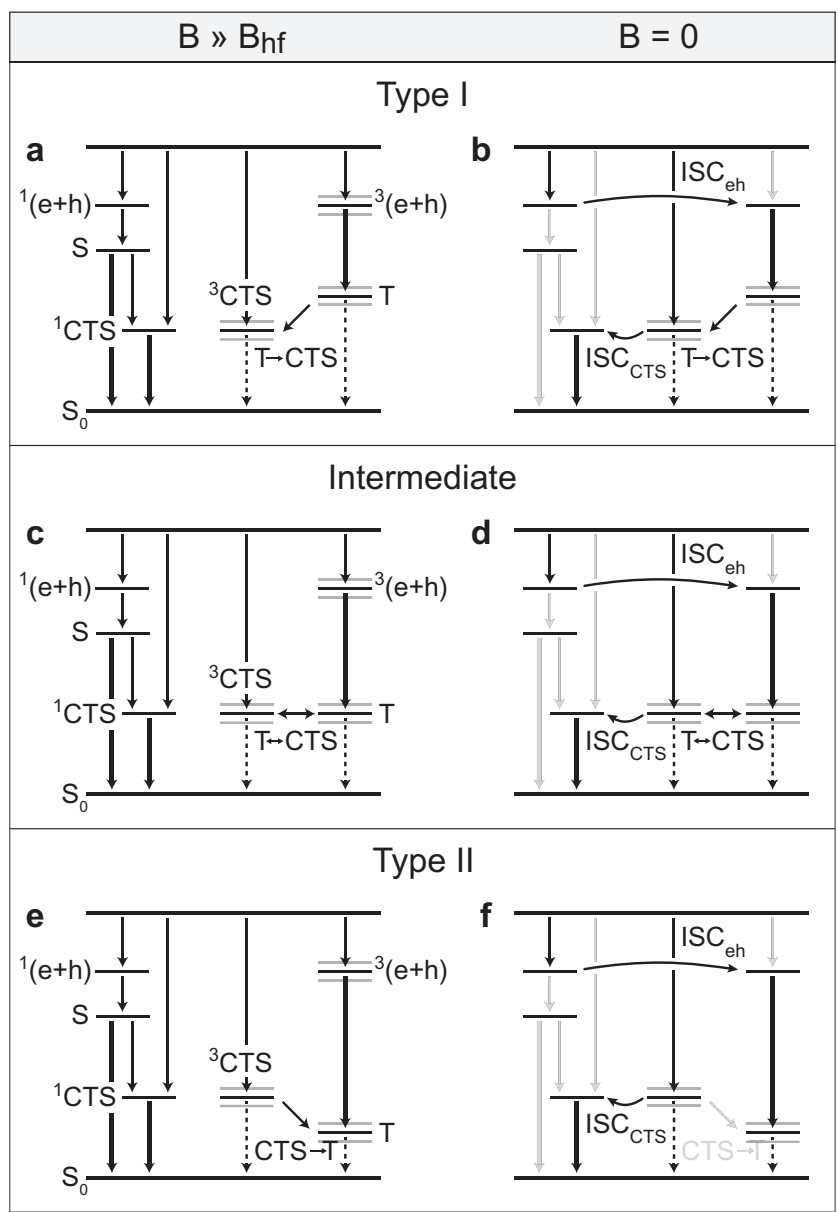

Fig. 2. Relevant spin dependent transitions for devices with different triplet exciton-charge transfer state (CTS) energy alignments. The introduction of CTS creates additional paths to the ground state. Three kinds of devices are depicted: the triplet exciton lies higher in energy than the CTS (Type I), both states are energetically aligned or the triplet exciton lies lower in energy than the CTS (Type II). The singlet and triplet CTS can also mix due to hyperfine fields, as

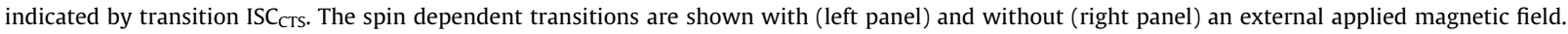

number of triplet excitons in the device by process $\mathrm{T} \rightarrow \mathrm{CTS}$ and this reduction is independent of the magnetic field. As a result, introducing CTS in device type I leads to a reduction of the MFE. In the intermediate case, the increase in the number of triplet excitons by process ISC $_{\mathrm{eh}}$ is diminished by process $\mathrm{T} \leftrightarrow \mathrm{CTS}$, also leading to a reduction in the MFE.

Finally, type II devices will show a distinctly different behavior when introducing CTS. The CTS is basically an electron-hole pair on two different molecules and therefore the singlet and triplet CTS can also undergo mixing due to hyperfine fields, as indicated by process ISC $_{\text {CTs }}$ in Fig.2. The difference with electron-hole pairs in the polymer is that CTS directly recombines to the ground state, therefore, the triplet recombination will be slower than the singlet recombination due to spin selection rules. In the absence of a magnetic field, (Fig.2f), spin mixing of CTS decreases the number of triplet CTS back-transferred to triplet excitons if the singlet CTS recombination rate is larger than the back-transfer rate. Increasing the magnetic field leads to an increase of the number of triplet excitons as indicated by process CTS $\rightarrow \mathrm{T}$ in Fig.2e. Thus, increasing the magnetic fields leads to more triplet-polaron interactions and thereby a decrease in the total current, resulting in a negative MC. This magnetic field effect is governed by the hyperfine field strength experienced by CTS $\left(B_{\mathrm{hf}, \mathrm{CTS}}\right)$ rather than the electron-hole pair $\left(B_{\mathrm{hf}, \mathrm{eh}}\right)$. This field strength will be significantly lower as a result of the very small hyperfine field coupling that electrons experience on the fullerene sites due to the vanishing nuclear magnetic moment of ${ }^{12} \mathrm{C}$ [15]. Thus, type II devices can give rise to two opposing MFEs with distinctly different linewidths.

In summary, the introduction of additional states, whereby the triplet exciton density can be influenced, could be a valuable tool to study triplet-polaron interactions unraveling their role in OMAR.

\section{Methods}

\subsection{Materials}

In this work we studied the magnetic field effects on the current for devices consisting of different polymer-fullerene blends. We used a variety of blends, but this study will focus on blends consisting of poly[(2-methoxy-5-(3,7- 
dimethyloctyloxy))-1,4-phenylenevinylene] (MDMO-PPV) and a phenyl substituted poly (1,4-phenylenevinylene) semiconducting polymer called Super Yellow PPV (SY-PPV). Both polymers were blended with [6,6]-phenylC61-butyric acid methyl ester (PCBM) in different concentrations. In these devices, PPV acts as hole-conducting polymer and electron donor, whereas PCBM acts as electron acceptor and this blend is a well-known and extensively studied organic photovoltaic system [21]. The MDMO-PPV was purchased from American Dye Source Inc., the SY-PPV from Merck and the PCBM from Solenne B.V. and all materials were used as received.

\subsection{Device fabrication}

The devices were prepared on glass substrates with patterned indium tin oxide (ITO) anodes. After careful cleaning, followed by a UV-ozone treatment, a thin layer of poly (3,4-ethylenedioxythipophene):poly (styrenesulfonate) (PEDOT:PSS) was applied by spin coating. The MDMO-PPV, SY-PPV and PCBM were dissolved in orthodichlorobenzene, with a concentration of 10,8 and $20 \mathrm{mg} / \mathrm{ml}$ respectively, and stirred on a hot plate at $50{ }^{\circ} \mathrm{C}$ for at least $2 \mathrm{~h}$ after appropriate blending. The blends were spin coated at $1200 \mathrm{rpm}$ for $60 \mathrm{~s}$. Subsequently, the samples were transferred to a nitrogen filled glove box where the cathode, consisting of LiF and $\mathrm{Al}$, was evaporated in a high vacuum system $\left(\sim 10^{-7} \mathrm{mbar}\right)$. From this point on, the samples always remain in a dry nitrogen environment. The total junction stack thus consisted of ITO/PEDOT:PSS ( $60 \mathrm{~nm}) /$ blend $(\sim 80 \mathrm{~nm}) / \mathrm{LiF}(1 \mathrm{~nm}) /$ $\mathrm{Al}(100 \mathrm{~nm})$, where the blend is either MDMO-PPV $(1-x)^{-}$ $\mathrm{PCBM}_{x}$ or SY-PPV $(1-x)-\mathrm{PCBM}_{x}$ and with $x$ the PCBM concentration in wt.\%.

\subsection{Measurements}

Magnetic field effect measurements were performed in a cryostat that is attached to a glovebox with a dry nitrogen environment $\left(\left[\mathrm{O}_{2}\right]<0.3 \mathrm{ppm},\left[\mathrm{H}_{2} \mathrm{O}\right]<0.3 \mathrm{ppm}\right)$. The cryostat is placed between the poles of an electromagnet, which allows us to apply an external magnetic field up to $0.5 \mathrm{~T}$, with an accuracy of $50 \mu \mathrm{T}$. The devices were driven at a constant voltage $V$ using a Keithley 2400 Series SourceMeter. We measured the current $I$ through the device while sweeping the magnetic field $B$. From this measurement, after correction for the drift, the magnetoconductance $(\mathrm{MC})$ was calculated with $\mathrm{MC}(B)=[I(B)-I(0)] / I(0)$.

\subsection{Empirical lineshapes}

To analyze the magnetoconductance, we used the following fitting function:

$$
\begin{aligned}
\mathrm{MC}(B)= & \mathrm{LFE} \cdot f\left(B, B_{\mathrm{LFE}}, B_{m}, r\right)+\mathrm{HFE} \cdot B^{2} /\left(|B|+B_{\mathrm{HFE}}\right)^{2} \\
& +\mathrm{CTS} \cdot f\left(B, B_{\mathrm{CTS}}, B_{m}, r\right)
\end{aligned}
$$

The function $f\left(B, B_{\mathrm{LFE}}, B_{m}, r\right)$, with $f(B=0)=0$ and $f(B=$ $\infty)=1$, is explained in full detail in Ref. [22]. The function correctly describes the low field effect (LFE), including the ultra-small field effect, in pristine polymer devices, as is shown in the right panel of Fig. 3a. This empirical function allows us to separately extract the role of the intrinsic hyperfine field $\left(B_{\mathrm{LFE}}\right)$ and the extrinsic additional broadening $\left(B_{m}\right)$ induced by the microscopic mechanism. The function converges to a Lorentzian lineshape for $f\left(B_{m}=0\right)$ and a so-called non-Lorentzian for $f\left(B_{m} \gg 0\right)$. The USFE is incorporated by the parameter $r$ which describes the limit in which hopping of carriers is no longer slow compared to spin precession in the hyperfine fields. The accompanying high field effect (HFE), depicted in the left panel of Fig. 3a, is fitted with non-Lorentzian lineshape and has a characteristic linewidth $B_{\mathrm{HFE}}$. Given the similarity of the magnetic field effect induced by charge transfer states compared to the LFE, we will use the same empirical function $f$, but using a necessarily different hyperfine field parameter $\left(B_{\mathrm{CTS}}\right)$.

In the fitting procedure, to reduce the number of fitting parameters, the intrinsic hyperfine strength $\left(B_{\mathrm{LFE}}\right)$, extrinsic additional broadening $\left(B_{m}\right)$ and the USFE parameter $(r)$ are determined for the pristine polymer devices and these values are then used for the doped devices. The intrinsic hyperfine strength of the CTS ( $\left.B_{\mathrm{CTS}}\right)$ and the high field effect linewidth $\left(B_{\mathrm{HFE}}\right)$ are determined in a global fit. The amplitudes of the magnetic field effects (LFE, HFE and CTS) can vary with composition and voltage.

\section{Results and discussion}

\subsection{Magnetic field effects}

To investigate the influence of additional states on magnetic field effects in the current, we have systematically measured the magnetoconductance as a function of magnetic field $B$, bias voltage $V$ and PCBM concentrations $x$ for two different polymer-fullerene blends. Typical results for three different concentrations $x$ are shown in Figs. 4 and 5 for MDMO and SY blends respectively. Both pristine devices (Figs. 4a, b, and 5a, b), show a similar lineshape with a positive LFE accompanied by a positive HFE and a USFE. When increasing the PCBM content, we observe a drastic decrease in amplitude of the MFEs. Although the LFE and HFE still show a similar shape for both polymers (Figs. 4c, e and 5c, e), the lineshape around zero applied field is completely different, as is clearly visible in Figs. $4 \mathrm{~d}$, f and $5 \mathrm{~d}$, f. We conjecture that the drastic decrease in amplitude as well as the remarkable differences in lineshape around zero applied field are caused by the introduction of CTS and their energetic alignment with respect to the triplet exciton in the polymer host. This conjecture will be put on more solid ground in the remainder of this article.

\subsection{Lineshapes analysis}

We performed a quantitative analysis by fitting the $\mathrm{MC}(B)$ data for all compositions and voltages with a superposition of a LFE (including USFE), a HFE and a contribution from the CTS (refer to the Methods section for a complete description). Thus, for each measurement, we get an amplitude of the LFE, HFE and CTS, and also the intrinsic hyperfine field scales ( $B_{\mathrm{LFE}}$ and $B_{\mathrm{CTS}}$ ), as well as the 


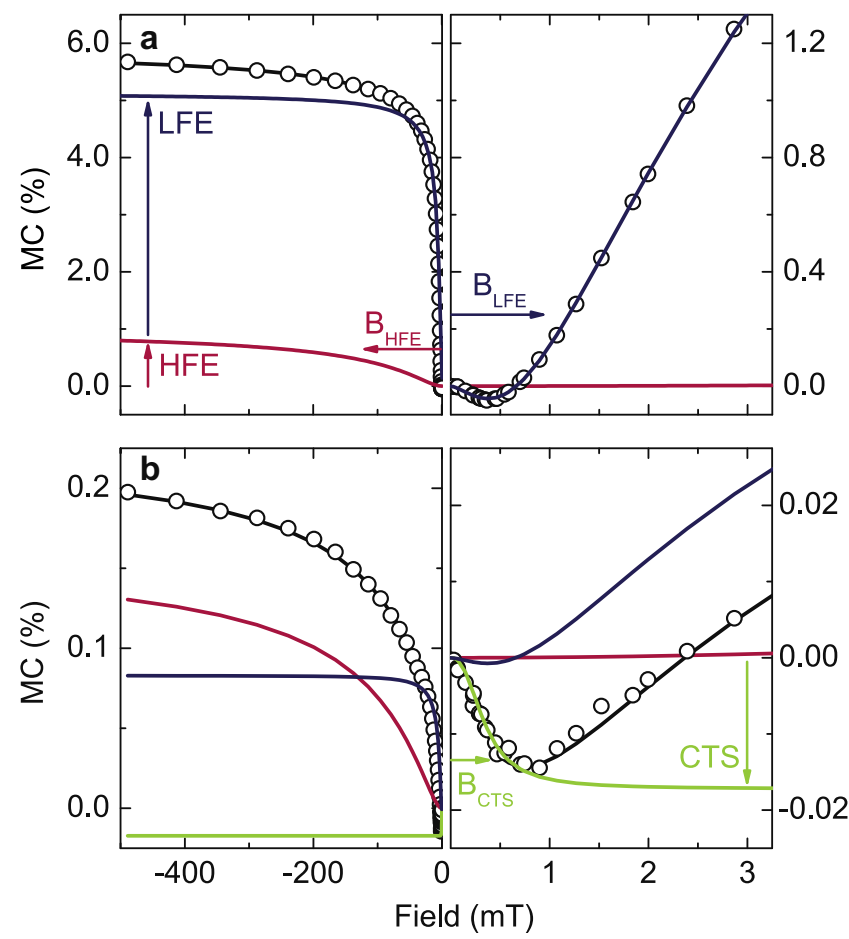

Fig. 3. (a) Magnetoconductance as a function of magnetic field for a bias voltage of $3.0 \mathrm{~V}$ for a pristine SY-PPV device. The solid line is a fit using the empirical lineshape, where the low (LFE) and high magnetic field (HFE) contributions are separately depicted. The magnitude of LFE and HFE and their corresponding linewidth parameters $\left(B_{\mathrm{LFE}}\right.$ and $B_{\mathrm{HLFE}}$ ) are also shown. (b) Magnetoconductance for a $1 \mathrm{wt}$ \% doped SY-PPV device at $3.0 \mathrm{~V}$. The MC clearly shows an additional low field contribution, which is included in Eq. (1) with an amplitude CTS and linewidth $B_{\text {CTS }}$.

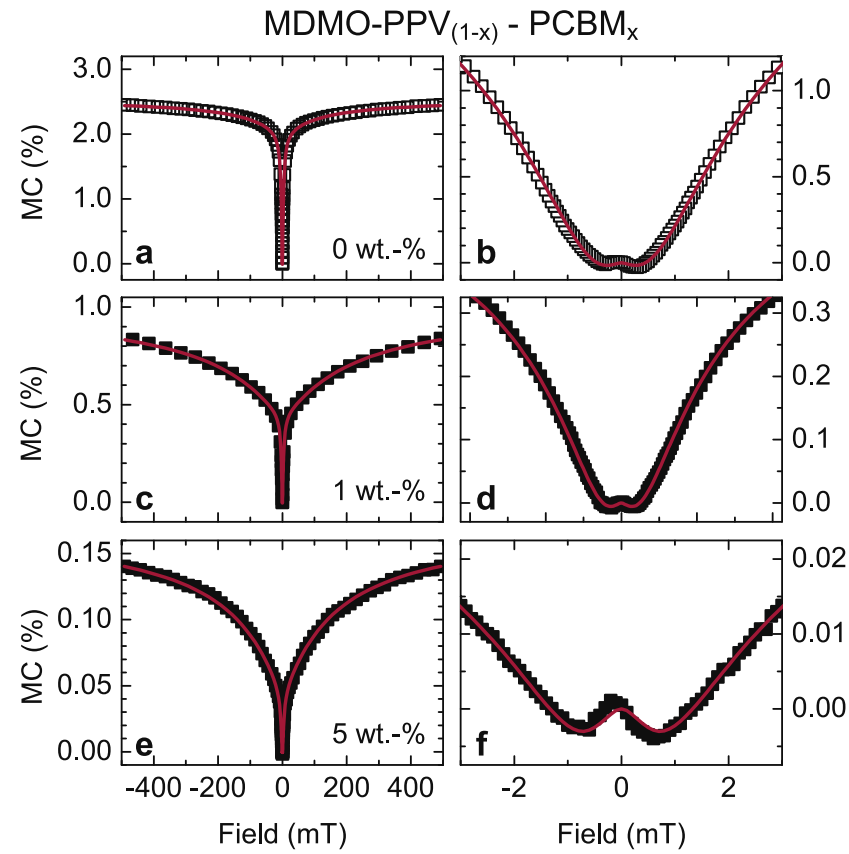

Fig. 4. Magnetoconductance as a function of magnetic field at $3.0 \mathrm{~V}$ for (a, b) a pristine, (c, d) 1 wt.\% doped and (e, f) 5 wt.\% doped MDMO-PPV device. The solid line is a fit using Eq. (1). The right panels (b, d, f) show a zoom around zero applied field.

linewidth of the HFE $\left(B_{\mathrm{HFE}}\right)$. The extracted parameters are shown in Fig. $6 a$ and $b$ present the amplitude of the MFEs in MDMO and SY blends respectively, while the linewidths are shown in Fig. $6 \mathrm{c}$ and d. We observe pronounced trends in the amplitudes of the MFEs, whereas the linewidths are approximately constant over the whole doping range 


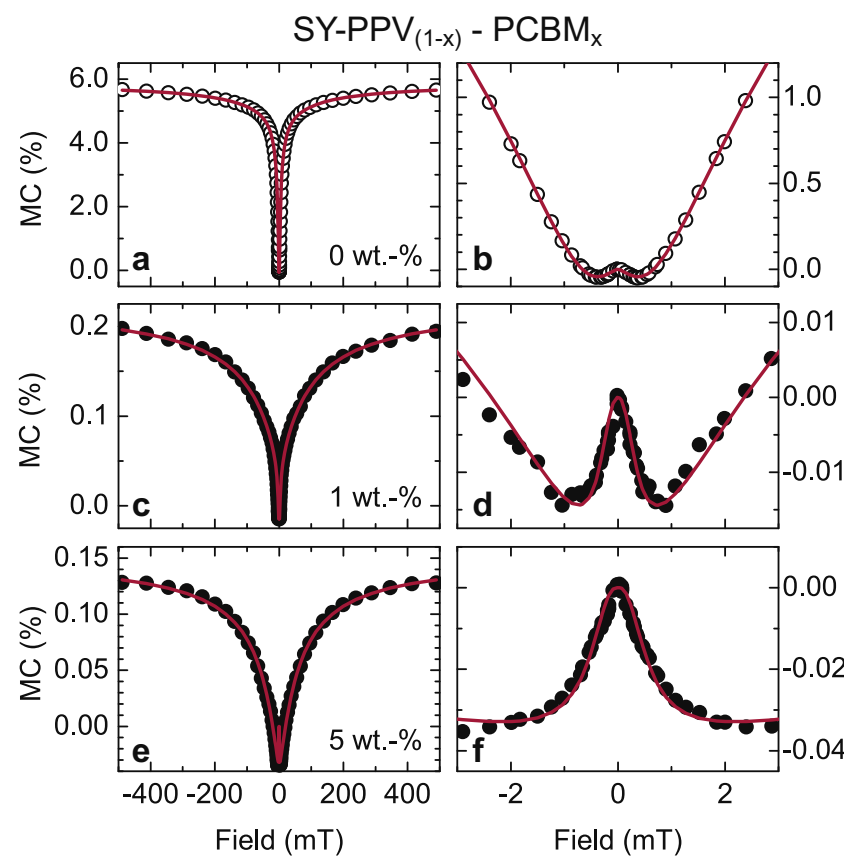

Fig. 5. Magnetoconductance as a function of magnetic field at $3.0 \mathrm{~V}$ for (a, b) a pristine, (c, d) 1 wt.\% doped and (e, f) 5 wt.\% doped SY-PPV device. The solid line is a fit using Eq. (1). The right panels (b, d, f) show a zoom around zero applied field.
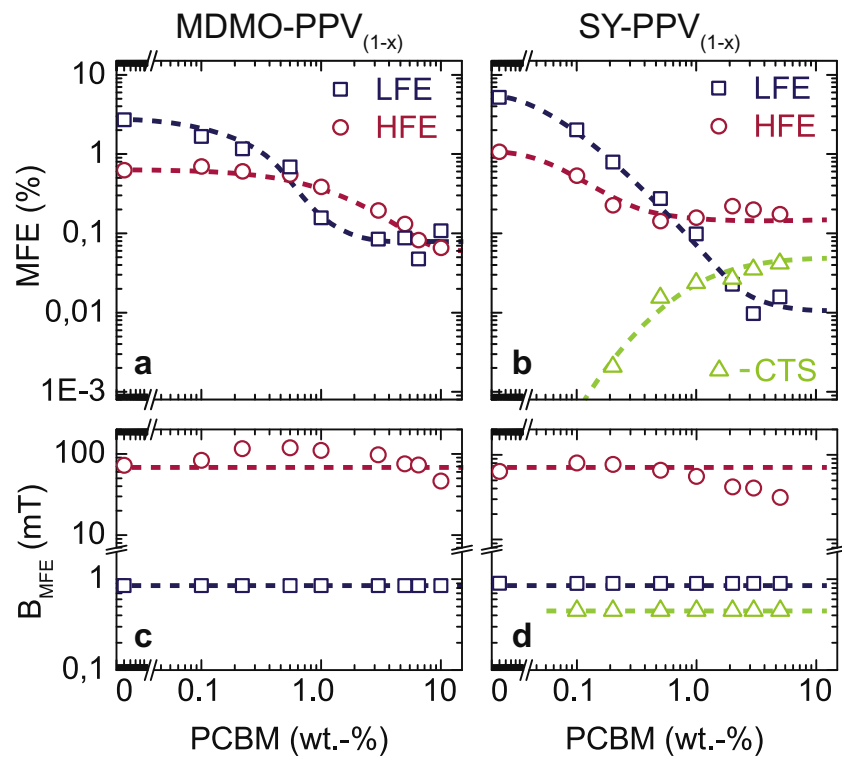

Fig. 6. Global trends of the magnetic field effects in the current (MFE) as a function of PCBM content, where the left panels (a, c) show the results for the MDMO-PPV device and the right panels (b, d) the SY-PPV device. The top panels (a, b) show the amplitude of the magnetic field effects, whereas the bottom panels (c, d) indicate the corresponding linewidths. The dashed lines provide a guide to the eye.

$\left(B_{\mathrm{LFE}}=0.85 \pm 0.10 \mathrm{mT}, B_{\mathrm{HFE}}=70 \pm 25 \mathrm{mT}\right.$ and $B_{\mathrm{CTS}}=0.45$ $\pm 0.05 \mathrm{mT})$. We previously stated that triplet-polaron interactions are the dominant underlying mechanism for the magnetic field effects in the current in our devices. The positive sign of the LFE and HFE, and the corresponding linewidths are consistent with this interpretation.

\subsection{Effect of additional states}

We will continue our discussion by examining the triplet exciton-CTS alignment. In both polymer-fullerene blends, the magnetic field effects caused by triplet-polaron interactions (LFE, HFE) are quenched by more than an 
order of magnitude by adding only a few wt.\% PCBM. However, in the SY-PPV blends, an additional MFE contribution arises when the PCBM concentration is increased. This contribution has the opposite sign as the LFE and the linewidth is significantly smaller. We therefore attribute this MFE to the CTS and assign the SY blend as type II, where the triplet exciton lies lower in energy than the CTS. The absence of an additional MFE caused by the CTS in MDMO-PPV blends, gives rise to a classification as type I. To confirm this hypothesis, we need to know the exact energy levels. However, only few reports about the energy alignments of CTS and triplet excitons in low ( $\leqslant 20 \mathrm{wt} . \%$ ) concentration polymer-fullerene blends are available in the literature [23-26]. Triplet energies in conjugated polymers, however, have been studied intensively [27-29]. From the literature, we can conclude that the triplet exciton and CTS in MDMO-PPV:PCBM blends are approximately aligned (1.40 eV [19] and 1.40-1.55 eV [23,25] respectively), whereas the triplet energy in SY-PPV lies slightly lower in energy (1.30 eV [30]). This is in agreement with our assignment. To further explore the effects of energy alignments on the MFEs, one would like to study devices with more pronounced differences in triplet exciton CTS energies. Therefore, we have identified the polymers PF10TBT $\left(E_{\mathrm{T}}=1.35 \mathrm{eV}\right.$ [24] and $E_{\mathrm{CTS}}=1.60 \mathrm{eV}$ [24]) and P3HT ( $E_{\mathrm{T}}=1.40$ [19] and $E_{\mathrm{CTS}}=0.90 \mathrm{eV}$ [31]) mixed with PCBM as good candidates.

\section{Conclusion}

In conclusion, in this article we presented a proof of concept study in which we tune triplet-polaron interactions in our devices by deliberately doping with fullerene which influences the triplet exciton density. We have systematically investigated the magnetic field effects on the current for two different polymer-fullerene blends. Using a quantitative analysis of the lineshapes we are able to extract the amplitudes and linewidths of the MFEs. We show that distinct changes in the lineshapes between the two polymerfullerene blends are correlated to the energetic alignment of the triplet exciton and CTS in the blends. Our findings enable a novel method to engineer the OMAR effect at ultra-small magnetic fields and, moreover, investigate the energetic alignment of excited states in organic semiconductors.

\section{Acknowledgements}

This work was supported by the Dutch Technology Foundation STW via the NWO VICI Grant "Spin Engineering in Molecular Devices" (Project No. 06628) and NWO NANO Grant "Chasing the spin in organic spintronics" (Project No. 11424).

\section{References}

[1] J. Kalinowski, M. Cocchi, D. Virgili, P. Di Marco, V. Fattori, Magnetic field effects on emission and current in Alq3-based electroluminescent diodes, Chem. Phys. Lett. 380 (2003) 710-715.

[2] T.L. Francis, O. Mermer, G. Veeraraghavan, M. Wohlgenannt, Large magnetoresistance at room temperature in semiconducting polymer sandwich devices, New. J. Phys. 6 (2004) 185.
[3] Ö. Mermer, G. Veeraraghavan, T. Francis, M. Wohlgenannt, Large magnetoresistance at room-temperature in small-molecular-weight organic semiconductor sandwich devices, Solid State Commun. 134 (2005) 631-636.

[4] V. Prigodin, J. Bergeson, D. Lincoln, A. Epstein, Anomalous room temperature magnetoresistance in organic semiconductors, Synth. Met. 156 (2006) 757-761.

[5] P. Desai, P. Shakya, T. Kreouzis, W.P. Gillin, N.A. Morley, M.R.J. Gibbs, Magnetoresistance and efficiency measurements of $\mathrm{Alq}_{3}$-based OLEDs, Phys. Rev. B 75 (2007) 094423.

[6] P.A. Bobbert, T.D. Nguyen, F.W.A. van Oost, B. Koopmans, M. Wohlgenannt, Bipolaron mechanism for organic magnetoresistance, Phys. Rev. Lett. 99 (2007) 216801.

[7] B. Hu, Y. Wu, Tuning magnetoresistance between positive and negative values in organic semiconductors, Nat. Mater. 6 (2007) 985-991.

[8] F.J. Wang, H. Bässler, Z. Valy Vardeny, Magnetic field effects in piconjugated polymer-fullerene blends: evidence for multiple components, Phys. Rev. Lett. 101 (2008) 236805.

[9] W.P. Gillin, S. Zhang, N.J. Rolfe, P. Desai, P. Shakya, A.J. Drew, T. Kreouzis, Determining the influence of excited states on current transport in organic light emitting diodes using magnetic field perturbation, Phys. Rev. B 82 (2010) 195208.

[10] A.J. Schellekens, W. Wagemans, S.P. Kersten, P.A. Bobbert, B. Koopmans, Microscopic modeling of magnetic-field effects on charge transport in organic semiconductors, Phys. Rev. B 84 (2011) 075204.

[11] S. Kersten, A. Schellekens, B. Koopmans, P. Bobbert, Magnetic-field dependence of the electroluminescence of organic light-emitting diodes: a competition between exciton formation and spin mixing, Phys. Rev. Lett. 106 (2011) 197402.

[12] M. Cox, P. Janssen, F. Zhu, B. Koopmans, Traps and trions as origin of magnetoresistance in organic semiconductors, Phys. Rev. B 88 (2013) 035202.

[13] C.E. Ambe, Y. Wakikawa, T. Ikoma, Thermal annealing effects on the photocarrier dynamics in PCBM films, Bull. Chem. Soc. Jpn. 86 (2013) $1051-1058$.

[14] T.D. Nguyen, B.R. Gautam, E. Ehrenfreund, Z.V. Vardeny, Magnetoconductance response in unipolar and bipolar organic diodes at ultrasmall fields, Phys. Rev. Lett. 105 (2010) 166804.

[15] P. Janssen, M. Cox, M. Kemerink, M. Wienk, B. Koopmans, Tuning organic magnetoresistance in polymer-fullerene blends by controlling spin reaction pathways, Nat. Commun. 4 (2013) 2286 .

[16] F.L. Bloom, M. Kemerink, W. Wagemans, B. Koopmans, Sign inversion of magnetoresistance in space-charge limited organic devices, Phys. Rev. Lett. 103 (2009) 066601.

[17] M. Cox, P. Janssen, S.H.W. Wouters, E.H.M. van der Heijden, M. Kemerink, B. Koopmans, The influence of device physics on organic magnetoresistance, Synth. Met. 173 (2013) 10-15.

[18] C. Deibel, T. Strobel, V. Dyakonov, Role of the charge transfer state in organic donor-acceptor solar cells, Adv. Mater. 22 (2010) 40974111.

[19] D. Veldman, S.C.J. Meskers, R.A.J. Janssen, The energy of chargetransfer states in electron donor-acceptor blends: insight into the energy losses in organic solar cells, Adv. Funct. Mater. 19 (2009) 1939-1948.

[20] T.D. Nguyen, G. Hukic-Markosian, F. Wang, L. Wojcik, X.-G. Li, E. Ehrenfreund, Z.V. Vardeny, Isotope effect in spin response of piconjugated polymer films and devices, Nat. Mater. 9 (2010) 345352.

[21] C. Deibel, V. Dyakonov, Polymer-fullerene bulk heterojunction solar cells, Rep. Prog. Phys. 73 (2010) 096401.

[22] W. Wagemans, P. Janssen, A.J. Schellekens, F.L. Bloom, P.A. Bobbert, B. Koopmans, The many faces of organic magnetoresistance, SPIN 01 (2011) 93.

[23] J. van Duren, X. Yang, J. Loos, C. Bulle-Lieuwma, A. Sieval, J. Hummelen, R. Janssen, Relating the morphology of poly(pphenylene vinylene)-methanofullerene blends to solar-cell performance, Adv. Funct. Mater. 14 (2004) 425-434.

[24] D. Veldman, I. Özlem, S. Meskers, J. Sweelssen, M. Koetse, S. Veenstra, J. Kroon, S. van Bavel, J. Loos, R. Janssen, Compositional and electric field dependence of the dissociation of charge transfer excitons in alternating polyfluorene copolymer-fullerene blends, J. Am. Chem. Soc. 130 (2008) 7721-7735.

[25] F. Piersimoni, S. Chambon, K. Vandewal, R. Mens, T. Boonen, A. Gadisa, M. Izquierdo, S. Filippone, B. Ruttens, J. D’Haen, N. Martin, L. Lutsen, D. Vanderzande, P. Adriaensens, J.V. Manca, Influence of fullerene ordering on the energy of the charge-transfer state and 
open-circuit voltage in polymer:fullerene solar cells, J. Phys. Chem. C 115 (2011) 10873-10880.

[26] S. Braun, W.R. Salaneck, M. Fahlman, Energy-level alignment at organic/metal and organic/organic interfaces, Adv. Mater. 21 (2009) 1450-1472.

[27] A.P. Monkman, H.D. Burrows, L.J. Hartwell, L.E. Horsburgh, I. Hamblett, S. Navaratnam, Triplet energies of pi-conjugated polymers, Phys. Rev. Lett. 86 (2001) 1358-1361.

[28] A. Khler, D. Beljonne, The singlet-triplet exchange energy in conjugated polymers, Adv. Funct. Mater. 14 (2004) 11-18.
[29] A. Köhler, H. Bässler, Triplet states in organic semiconductors, Mater. Sci. Eng. R - Rep. 66 (2009) 71-109.

[30] V. Jankus, E.W. Snedden, D.W. Bright, V.L. Whittle, J.A.G. Williams, A. Monkman, Energy upconversion via triplet fusion in super yellow PPV films doped with palladium tetraphenyltetrabenzoporphyrin: a comprehensive investigation of exciton dynamics, Adv. Funct. Mater. 23 (2013) 384-393.

[31] Y. Kim, S. Choulis, J. Nelson, D. Bradley, S. Cook, J. Durrant, Composition and annealing effects in polythiophene/fullerene solar cells, J. Mater. Sci. 40 (2005) 1371-1376. 